\title{
Un debate entre lo personal y lo público: la interdicción como pretexto
}

\section{A debate between the personal and the public spheres: the interdiction as pretext}

\begin{abstract}
Resumen
La apuesta de este texto es poner en entredicho la figura de interdicción como un elemento netamente legal y constituirla como un procedimiento reproductor de representaciones sociales y prácticas que intervienen directamente en el ejercicio de la autonomía de las personas con discapacidad, tanto en la esfera pública como en la privada, visibilizando los supuestos ontológicos que subyacen a esta práctica y los efectos que son percibidos por las personas con discapacidad a partir de sus narrativas. Se expone la conceptualización de la capacidad jurídica a la luz de los modelos de discapacidad, los abordajes desde la legislación colombiana, las voces mostradas en la literatura científica de personas con discapacidad intelectual respecto a sus procesos de toma de decisiones en salud sexual y reproductiva y la interdependencia como una posible alternativa a un modelo en el que las personas con discapacidad intelectual sean reconocidas como sujetos de derechos.
\end{abstract}

\section{Palabras clave}

Interdicción, público, privado, interdependencia, dependencia, autonomía, discapacidad intelectual, capacidad jurídica.

\begin{abstract}
The aim of this text is to call into question the interdiction figure as a purely legal element and to constitute it as a reproductive procedure of social representations and practices that directly intervene in the exercise of the autonomy of persons with disabilities, both in the public sphere and in the private, making visible the ontological assumptions that underlie this practice and the effects that are perceived by people with disabilities from their narratives. The conceptualization of the legal capacity is exposed in the light of disability models, the approaches from the Colombian legislation, the voices shown in the scientific literature of people with intellectual disability regarding their decision making processes in sexual and reproductive health and interdependence as a possible alternative to a model in which people with intellectual disabilities are recognized as subjects of rights.
\end{abstract}

\section{Keywords}

Interdiction, public, private, interdependence, dependence, autonomy, disability, legal capacity.

\author{
Melissa Stefania Cruz \\ Puerto \\ <mscruzp@unal.edu.co> \\ Universidad de Nacional de \\ Colombia. Colombia
}

\section{Edna Rocío Rincón León <errincon|@unal.edu.co>}

Universidad de Nacional de Colombia. Colombia

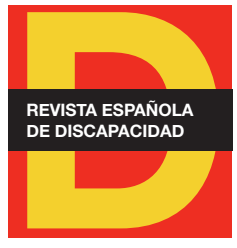

Para citar:

Cruz, M. S y Rincón, E. R. (2018): “Un debate entre lo personal y lo público: la interdicción como pretexto". Revista Española de Discapacidad, 6 (I): 75-90.

Doi: <https://doi.org/10.5569/23405104.06.01.04>

Fecha de recepción: 19-02-2017 Fecha de aceptación: 11-01-2018 


\section{Introducción}

La interdicción y la toma de decisiones de las personas con discapacidad son temas clave en los debates que se generan al interior de los movimientos de vida independiente en varios lugares del mundo, en los cuales se logra percibir que las prácticas alrededor de estos tópicos son producto de un entramado de relaciones de poder y dominación basadas en representaciones sociales históricamente construidas. Su relevancia se manifiesta en actitudes paternalistas que esconden la falta real de reconocimiento de la condición humana y que van desde la imposibilidad de tomar decisiones informales en la vida cotidiana, por ejemplo sobre la alimentación, el vestuario, el cuerpo o la sexualidad, hasta aspectos formales como los asuntos económicos, bancarios o consentimientos para tratamientos médicos. Partiendo de la premisa de que existe una ausencia de claridad para determinar los límites entre lo privado y lo público, y que estas esferas con frecuencia se sobreponen en diferentes ámbitos, se presenta la interdicción como un procedimiento que va más allá del ámbito público y legal, convirtiéndose en una práctica reproductora de representaciones sociales y experiencias que intervienen directamente en la trayectoria de vida de las personas con discapacidad y sus familias.

La apuesta de este texto es poner en entredicho la figura de interdicción como un elemento netamente legal y constituirla como un procedimiento reproductor de representaciones sociales y prácticas que intervienen directamente en el ejercicio de la autonomía de las personas con discapacidad, visibilizando los supuestos ontológicos que subyacen a esta práctica y los efectos que son percibidos por las personas con discapacidad a partir de sus narrativas. Para tal fin, comenzaremos exponiendo una aproximación a los conceptos que subyacen a la implementación de esta práctica desde los modelos de la discapacidad y en el contexto colombiano, teniendo como referencia el marco normativo; en segundo lugar, se introducirán los aspectos encontrados en la literatura que ponen de manifiesto las voces de las personas con discapacidad que hacen evidente una distancia entre sus vivencias reales y el modelo social planteado por la Convención y, finalmente, se expondrá el concepto de interdependencia como una posible línea de fuga para la implementación real de un modelo de apoyo en toma de decisiones que respete la autonomía de las personas con discapacidad como componente fundamental de la dignidad humana.

\section{La construcción del sujeto interdicto e incapaz en relación con las concepciones de la discapacidad}

Es importante comenzar aclarando que la discapacidad no da cuenta de un concepto único e inequívoco, sino que por el contrario es un producto histórico que ha dado cuenta de las demandas sociales y políticas de diferentes épocas y comunidades. Como concepto, la discapacidad ha tomado diferentes niveles interpretativos de cómo representar la discapacidad, los cuales se han organizado en modelos explicativos. Existe un consenso en la existencia de tres modelos de la discapacidad: el de prescindencia, el médico y el social.

El modelo de prescindencia marcó la historia de la discapacidad desde la Antigüedad hasta principios de la Ilustración en el mundo occidental. Este modelo concibe la discapacidad como un problema personal que 
es padecido por un cierto número de personas y que muchas veces es el resultado de faltas cometidas, pecado o maldad, relacionando directamente la discapacidad con juicios de valor negativos desde marcos normativos sociales y religiosos.

Toboso y Arnau (2008) plantean la división del modelo de prescindencia en dos submodelos: el eugenésico y el de la marginación. La eugenesia nace como un fenómeno social y se establece como una técnica de control poblacional con el fin de asegurar el nacimiento de seres ideales frente a los estándares éticos y económicos de la época. Dicho movimiento se basó en "teorías de la degeneración, explicaciones hereditaristas de la desviación, y campañas para eliminar las poblaciones indeseables y no aptas (por ejemplo, los inmigrantes, los delincuentes, los pobres y los débiles mentales)" (Carlson, 2010: 60), generando una deshumanización de las personas que no cumplían con dicha imagen ideal al ser puestos al nivel de los animales y desprovistos de sus derechos a través de acciones de control como la institucionalización, la esterilización, la pérdida de capacidad legal y jurídica y el asesinato sistemático. Desde el submodelo de la marginación las personas con discapacidad son percibidas como objetos de compasión sin ningún valor social: son relegadas a lugares y roles sociales marginales, estimando negativamente su aporte a la sociedad desde parámetros económicos y productivos (Palacios y Romañach, 2006). Como consecuencia, las personas con discapacidad son consideradas como innecesarias, "la sociedad decide prescindir de ellas ya sea a través de políticas eugenésicas o a través de la marginación” (Benavides, 2013: 18). Desde este modelo las personas con discapacidad no eran consideradas como ciudadanas y en muchas ocasiones tampoco como seres humanos, por lo cual no existía una concepción cercana para su capacidad legal o autonomía.

Por otro lado, el modelo médico nace de la mano con una visión rehabilitadora de la discapacidad que la comprende como un problema individual producto de una condición de salud que no tiene ninguna relación con factores externos, por el contrario, se trata de un funcionamiento anormal de un órgano, extremidad, función corporal o mental. Desde esta visión el rol y valor social de las personas con discapacidad queda vinculado a diversos procesos rehabilitadores con el fin de normalizar al sujeto. A diferencia del anterior modelo, las personas con discapacidad pueden ser poseedoras de capacidad jurídica en tanto culminen de manera exitosa su proceso de rehabilitación/normalización.

Estas concepciones desembocan en el desarrollo de un punto de vista paternalista del -así considerado- incapaz, se adjudica a él la imposibilidad del ejercicio de la autonomía por no contar con habilidades cognitivas o emocionales para prever las consecuencias de sus actos. La acción principal contemplada desde el derecho es la representación legal y prosigue con lo que Fernández de Bujan (2012) denomina la incapacitación, que consiste en limitar la capacidad de obrar de una persona, bien sea por demencia, analfabetismo o impulsividad para gastar el patrimonio familiar a partir de unos parámetros preestablecidos de normalidad y capacidad. Desde este modelo de corte paternalista la capacidad jurídica para las personas con discapacidad es negada y sustituida por la figura de interdicción, con base en argumentos de protección e incapacidad desde parámetros legales y médicos.

Simultáneamente a esta secuencia de eventos, las personas con discapacidad comienzan a reconocerse como un grupo poblacional sistemáticamente excluido e inician procesos de movilización política y social en búsqueda del reconocimiento de sus derechos generando un cambio en la perspectiva de la discapacidad, ya que a partir del lema "nada acerca de nosotros sin nosotros", luchan "por mejorar [su] calidad de vida, asegurar la accesibilidad al medio físico y social y el derecho a una vida independiente" (Valencia, 
2014: 12). Al entenderse la discapacidad como el producto de la relación del individuo con su entorno físico, cultural y social, se deja de lado su conceptualización como un problema de salud y tragedia personal y se comienza a reemplazar el discurso de la institucionalización por el discurso de la inclusión social.

Un fruto del advenimiento de estos movimientos sociales es la adopción de la Convención Internacional sobre los Derechos de las Personas con Discapacidad (CDPD) que produce un cambio significativo en el paradigma de capacidad jurídica, que pretende pasar de la sustitución a la toma de decisiones al apoyo en dicho proceso (Lorenzo et al., 2008).

En este contexto, la capacidad jurídica es vista como la manifestación de la voluntad y preferencias de una persona, que implica ser portador de derechos y obligaciones, y la posibilidad de ejercerlos con los apoyos que se requieran para tal fin. Se consolida así como un derecho inherente al ser humano cuya capacidad mental se comprende como la aptitud para adoptar decisiones que varía en función de muchos factores personales y contextuales en contraposición a un fenómeno objetivo susceptible de ser medido; los déficits supuestos o reales en esta capacidad no pueden ser justificación para negar la capacidad jurídica (Jaime, 2015: 21).

Inclusion International ha identificado la autodeterminación como uno de los principios fundamentales dentro del movimiento social y político de personas con discapacidad, ya que representa el hecho de que "todas las personas deben tener el control de las decisiones que afectan su vida" (2014: 14). La capacidad jurídica desde aquí no se entiende solo desde un marco netamente legal y jurisprudencial, se trata de una contextualización de las condiciones, itinerarios y trayectorias de vida de las personas con discapacidad a partir de su capacidad de elección desde las cosas más privadas como la sexualidad hasta aspectos más generales como la participación política, esto con el fin de garantizar el pleno goce de sus derechos.

Este cambio de perspectiva de la concepción de capacidad legal es visible en el Informe Mundial Sobre el Derecho a Decidir, que afirma que:

"[...] Con apoyo, todas las personas con discapacidad intelectual son capaces de tomar decisiones y tener control en su vida. El apoyo puede adoptar muchas formas: la disponibilidad de información en lenguaje sencillo, asistencia para entender las opciones y consecuencias de las decisiones, tiempo adicional para realizar cada elección, etc. En el caso de las personas con mayores necesidades de apoyo o dificultades para comunicarse, el apoyo podría consistir en una red de personas que manifiesten y expongan las decisiones basándose en la voluntad e intención de la persona" (Inclusion International, 2014: 14).

\section{Contexto colombiano}

En el contexto colombiano es posible encontrar desarrollos legales que mudan históricamente y permiten leer entre líneas una transformación de la concepción de la persona con discapacidad y la capacidad legal. Sin embargo, un continuo que se presenta es la presencia de argumentos y concepciones desde el modelo 
de la prescindencia relacionándola con la exclusión y la eugenesia, negando su capacidad de decisión en situaciones cotidianas y privadas como la sexualidad y la procreación.

Para el Código Civil Colombiano, sancionado en 1873, los ciudadanos somos comprendidos como capaces o incapaces. Se parte del supuesto de que "todas las personas son capaces para ejercer sus derechos civiles hasta que un acto legal establezca lo contrario; en esta normativa se contemplan como incapaces el disipador, el demente, los ciegos, los sordomudos, las mujeres y los menores de edad" (Código Civil, 1873). Aparece la figura de interdicción que puede ser conceptualizada como "la sustracción de la capacidad jurídica de una persona por medio de la declaración de discapacidad mental absoluta" (Jaime, 2015: 14). Es un procedimiento que realiza un juez con el fin de asignar a un representante a aquellos incapaces que no pueden velar por sus propios intereses.

La capacidad jurídica tal como es entendida por el Código Civil implica dos escenarios: ser titular de derechos y obligaciones, y la aptitud para ponerlos en ejercicio obrando de acuerdo con la autonomía y la razón. Esta capacidad de acción es variable en el transcurso vital y puede tenerse o perderse si una acción judicial lo demanda (Benavides, 2013: 8).

Para Jaime (2015), esta perspectiva crea una relación de concomitancia entre la capacidad jurídica con la capacidad mental, esta última entendida como un fenómeno objetivo susceptible de ser medido. De acuerdo con este autor, en el año 2009 se aprueban dos leyes contradictorias referentes a la conceptualización de capacidad jurídica: la ley 1306 "por la cual se dictan normas para la protección de personas con discapacidad mental y se establece el régimen de la representación legal de incapaces emancipados", y la ley 1346 que aprueba la Convención sobre los Derechos de las Personas con Discapacidad. Esta contradicción genera situaciones claramente inconstitucionales desde la perspectiva de este autor; abriendo la posibilidad a prácticas como la interdicción o la anticoncepción quirúrgica sin autorización, que son violatorias de derechos desde la perspectiva de la Observación General del Comité sobre los derechos de las personas con discapacidad.

La ley 1306 de 2009 es la norma que rige la interdicción actualmente. Su implementación se ha reflexionado en varias sentencias de la Corte Constitucional que permiten evidenciar los conflictos derivados de las contradicciones ya enunciadas entre esta ley y un enfoque de derechos como el propuesto en la Convención. Estas sentencias más recientemente mudan hacia la perspectiva de apoyo en la toma de decisiones como es el caso, por ejemplo, de la Sentencia T740 de 2014.

"En el estudio de casos particulares, la Corte ha ido decantando las eventuales hipótesis en las que puede resultar admisible excepcionalmente la autorización del procedimiento de esterilización quirúrgica en menores en situación de discapacidad [...]. Así, ha encontrado (primera excepción) que si existe un riesgo a la vida de la paciente como consecuencia del embarazo y la imposibilidad de evitarlo eficazmente por otros medios, se preferirá salvaguardar la vida e integridad de la menor en condición de discapacidad siempre que esta, de manera reflexiva y consciente, no decida lo contrario. Esta subregla presupone los siguientes requisitos: (i) que la decisión sea consentida por la menor; (ii) que un grupo interdisciplinario certifique que la misma conoce y comprende las consecuencias de la intervención quirúrgica; (iii) que exista un concepto médico interdisciplinario que establezca que la operación es imprescindible para proteger su vida porque no exista otra alternativa; y (iv) que, en todo caso, se otorgue autorización judicial para garantizar el respeto de los derechos del menor, con especial énfasis en determinar la posibilidad 
para consentir o no el procedimiento médico. El otro caso (segunda excepción), lo constituye la circunstancia de discapacidad severa o profunda en la que puede presentarse la situación de inexistencia de capacidad para emitir consentimiento futuro, caso en el que parte de la jurisprudencia constitucional ha considerado que no se atenta contra el derecho a la autonomía del menor porque este no la puede ejercer, dado que el menor no comprende las implicaciones de la operación ni el significado de la maternidad o paternidad" (Corte Constitucional, 2014).

La ley 1306 de 2009 introduce los conceptos de discapacidad mental absoluta y discapacidad mental relativa, a partir de los cuales el juez otorga la figura de incapaz absoluto (no puede realizar o se le considera nulo cualquier acto voluntario con connotación jurídica) o incapaz relativo (quien puede contar con la validez jurídica de algunos de sus actos como casarse o administrar parte de su patrimonio, entre otras). La acción de interdicción está fundamentada en una protección de doble vía: intenta evitar que la persona con discapacidad se perjudique a sí misma o a los demás y proteger su integridad. Se habla aquí de personas con discapacidad mental en contraposición a aquellas que presentan discapacidad física, diferenciación que se establece a partir del juicio valorativo y profesional de profesionales de la ciencia médica.

Entre las medidas establecidas para la restricción de la capacidad jurídica se cuentan la interdicción para personas con discapacidad mental absoluta o la inhabilitación para el caso de discapacidad mental relativa. Esta restricción puede obtenerse de manera provisional o definitiva y se lleva a cabo ante los jueces de familia una vez exista dictamen de un experto. El ejercicio de deberes de los individuos con discapacidad intelectual (civiles, políticos, religiosos) queda restringido en su totalidad sin importar si dicho ejercicio tiene o no influencia en su estado de salud. Una vez son considerados como incapaces, la responsabilidad inicial se dirige a la familia o la persona designada como curador principal y el estado adquiere la función de vigilar a través de un juez de familia. En este marco procedimental, se ha determinado que figuras como la familia tienen el poder desde y frente a la ley para representar a la persona con discapacidad mental, creando figuras como la del curador, el administrador, el guardador y el consejero (Medina et al., 2009).

Este último aspecto en el que se considera a la familia como principal representante del así declarado incapaz a partir del proceso de interdicción resulta particularmente problemático, pues termina sometiendo a la persona a una dinámica en la que sus seres más allegados ejercen control sobre las decisiones. El criterio para determinar los límites de la injerencia del representante, así como la decisión sobre cuáles esferas de la vida de la persona son susceptibles de controlar, son aspectos puestos a merced del conocimiento y las representaciones sociales circundantes que haya apropiado la familia sobre la vida de la persona, ahora despojada de su autonomía mediante un sustento legal.

Para ejemplificar cómo es la puesta en marcha de la norma en la realidad, actualmente la capacidad de decisión en temas tan polémicos como la anticoncepción quirúrgica sin consentimiento por parte de la persona con discapacidad intelectual gira sobre el concepto de consentimiento orientado hacia el futuro que aparece en la sentencia C-131 de 2014 de la Corte Constitucional, la cual busca:

“[...] Proteger la decisión que mejor preserve la integridad de las condiciones físicas necesarias para que la persona que aún no cuenta con la autonomía suficiente para tomar decisiones sobre su propia vida y salud, pueda decidir cómo va a ejercer dicha libertad en el futuro" (p. 10).

Los supuestos que subyacen a esta forma de abordar el tema se basan en la capacidad que tienen las personas para decidir sobre aspectos presentes que pueden afectar a su futuro. En este caso se plantean 
las intervenciones quirúrgicas anticonceptivas como una circunstancia presente que afecta a la decisión futura de tener hijos o del número de hijos que se quieren tener. En lo referente a la esterilización quirúrgica de los menores en condición de discapacidad, dicha sentencia regula que en el caso de que la persona pueda otorgar su consentimiento futuro frente a dicha intervención, puede ejercer el derecho a decidir si realizarla o no. Si el sujeto no puede tomar dicha decisión, serán sus padres quienes deberán solicitar una autorización judicial para llevar a cabo la operación. Dicha capacidad de consentimiento futuro se determina a partir de los parámetros médicos y de las indicaciones de profesionales de la salud y será un juez ordinario quien adoptará las medidas que resulten necesarias para determinar si la persona con discapacidad intelectual tiene o puede llegar a tener el grado de autonomía suficiente para decidir acerca de la procedencia o no del mencionado procedimiento quirúrgico como se especifica en la sentencia la T-560A de 2007. Este procedimiento no es claro y puede variar a partir de la decisión de los jueces, ya que no se cuenta con un mecanismo o protocolo preestablecido para tal fin y muchas veces queda sometido a representaciones, estereotipos y estigmas que el personal judicial y médico mantiene sobre la discapacidad.

Referente a este tema el Comité sobre los derechos de las personas con discapacidad de la ONU examinó el informe inicial de Colombia (CRPD/C/COL/1) en sus sesiones $281^{a}$ y $282^{a}$ celebradas los días 23 y 24 de agosto de 2016 respectivamente, y planteó las siguientes observaciones relacionadas con la figura legal de la interdicción y sus implicaciones en diferentes esferas de la vida privada. El Comité expresa:

"[...] 52. Su preocupación por que las personas con discapacidad cuya capacidad jurídica se ve restringida debido a la declaratoria de interdicción no puedan contraer matrimonio ni formar una familia sin autorización judicial.

53. Insta al Estado parte a que derogue toda restricción que limite o impida a personas con discapacidad contraer matrimonio y formar una familia sobre la base del consentimiento previo de las parejas. Además le recomienda que tome medidas para prohibir explícitamente en la ley colocar a los niños fuera de sus familias sobre la base de la discapacidad, y garantizar mecanismos de apoyos disponibles en la comunidad para progenitores con discapacidad" (ONU, 2016).

En general, la Convención de la ONU invita a los miembros participantes a implementar un modelo de toma de decisiones con apoyo y eliminar la figura de la interdicción. Sin embargo, en países como Colombia y algunos latinoamericanos esta figura todavía se mantiene, reproduciendo imaginarios negativos sobre la discapacidad a partir de representaciones eugenésicas y de prescindencia que regularizan las violaciones contra los derechos de esta población.

Una de las posibles rutas, en el caso colombiano, es el proyecto de ley No más interdicción para las personas con discapacidad (2017), el cual se basa en la premisa de que el no reconocer la capacidad jurídica es una forma de limitar el ejercicio de la ciudadanía plena para cualquier persona. Este proyecto de ley busca eliminar la figura de la interdicción y cambiarla por un modelo de toma de decisiones con apoyo, a partir de la asistencia y el acompañamiento de redes sociales cercanas, estableciendo tres mecanismos de apoyo en la toma de decisiones: (1) los acuerdos de apoyo, (2) la adjudicación judicial de apoyos y (3) las directivas anticipadas. El primer mecanismo se realiza por escrito y es una decisión que se toma de forma conjunta entre la persona con discapacidad y aquellas que le van a brindar el apoyo. Este acuerdo se puede eliminar en cualquier momento por mutua decisión. El segundo mecanismo de apoyo plantea que en casos excepcionales un juez es quien realizará el proceso de adjudicación de apoyo a partir de un proceso de valoración técnico preestablecido, en 
los casos de personas que puedan saber que requieren apoyo pero no puedan reconocer el grado de este o que no tengan a ninguna persona que los apoye. El tercer modelo es una forma de apoyo formal por medio de la cual cualquier persona puede tomar decisiones anticipadas sobre su salud, finanzas o asuntos personales, las cuales quedarán por escrito y se deberán respetar y realizar como se preestablecieron.

Para Cuenca (2012: 22), una nueva concepción y regulación de la capacidad jurídica para las personas con discapacidad debe dejar de lados prácticas y normas que permitan la restricción de la capacidad y pasar a un modelo de reconocimiento de la igualdad de capacidad tanto jurídica como de obrar de todas las personas, sin excepción por discapacidad u otros factores. Este nuevo modelo debe plantearse a partir de dos premisas: la primera es que el hecho de que una persona cuente con medidas de apoyo para la toma de decisiones u obrar no supone limitaciones en su capacidad ni en su condición de ciudadanía, y la segunda es que se deben cumplir con las obligaciones de accesibilidad y ajustes razonables necesarios y suficientes para poder ejercer dicha capacidad.

\section{Del concepto a la realidad}

Pensar en los cambios normativos a nivel internacional como los sugeridos por el enfoque de derechos de la Convención puede sugerir un panorama alentador, sin embargo, una revisión de la literatura permite entrever una diferencia significativa entre tales aspectos formales y la realidad que enfrentan las personas con discapacidades. Como ya se ha mencionado, se trata de un continuo de imposiciones que van desde la decisión de casarse o tener familia hasta la negación del derecho a poseer propiedad privada y participar en espacios de decisión política. En la actualidad este grupo poblacional continúa enfrentando discriminación, asistencia inapropiada, problemas con la custodia de sus hijos y múltiples barreras sociales que impiden el libre desarrollo de este tipo de decisiones (McConnell, 2012).

A partir de la concepción de racionalidad, capacidad y del modelo eugenésico que subyace a estas acciones legales, se extrapola esta categoría igualando a las personas con discapacidad intelectual a eternos niños (asexuados) o a animales (con instintos desenfrenados) (López et al., 2010), cuya reproducción queda vetada, en parte con el objetivo de evitar abusos pero también para evitar que sus características biológicas se transmitan a nuevas generaciones. En esferas íntimas esto se traduce en representaciones sociales que implican la visión de su sexualidad como perversa, inexistente o anómala y la configuración de actitudes visibles en las prácticas cotidianas de las familias, actores educativos y del sector salud.

Con el ánimo de identificar las vivencias de las personas con discapacidad, se presentarán algunas voces presentes en la literatura nacional e internacional en relación con la capacidad de toma de decisiones respecto a temas como la afectividad y la sexualidad, poniendo énfasis en las dificultades de acceso a la información, las voces de las familias, desde la postura de lo personal como escenario político.

La sexualidad es uno de esos temas transversales a muchas discusiones sobre derechos que no es extraño dentro de los debates sobre autonomía y elección de las personas con discapacidad. Un grupo de artistas 
de Medellín con discapacidad intelectual manifiestan, por ejemplo, cómo sienten restringida su capacidad de acción y decisión respecto a su propia sexualidad debido a las prohibiciones de los padres y la ausencia de información sobre el tema. Sienten que el entorno selecciona la información que reciben, lo que implica un ejercicio de control sobre ellos. La concepción de sus familias de ellos como niños es un dispositivo de control que impera en lo legal y en lo cultural. Este aspecto es experimentado como una barrera al disfrute pleno de sus relaciones afectivas (Ramírez et al., 2015; Rojas et al., 2014).

Este control en la información a la que se accede es aún más crítico cuando se habla de orientaciones sexuales no normativas, como lo evidencia un estudio conducido por Stoffelen et al. (2013). A través de las experiencias de vida con personas con discapacidad intelectual homosexuales de Países Bajos, estos autores muestran cómo al intentar hablar de temas relacionados con el ejercicio de su sexualidad, las personas se encuentran con la negativa tanto de profesionales como de la familia e instituciones educativas.

En relación con las familias, un estudio conducido por Herazo (2015) en el contexto colombiano encuentra una estructura familiar rígida y sobreprotectora. Esta autora manifiesta como existe una relación de vital importancia entre las creencias familiares y la impronta de identidad en el sujeto. Adicionalmente, considera que la relación de sexualidad, identidad y proyecto de vida son aspectos importantes que configuran la subjetividad y posicionamiento en el mundo de la persona con discapacidad. En el análisis que adelanta de las narrativas de las madres de hijos con discapacidad es posible identificar una visión idealizada de la discapacidad intelectual, un posicionamiento de los hijos como personas en las que no hay una atribución de intencionalidad sexual ni afectiva. Para algunas madres sus hijos son considerados eternos niños, lo que los ubica en el lugar de seres dependientes. Las madres entrevistadas por esta autora no parecen tener un cuestionamiento claro de la forma en la que se imparte información sobre la sexualidad y con respecto al proyecto de vida. Por el contrario, se perpetúa la visión de dependencia, lo que impide un cuestionamiento sobre las posibilidades de autorrealización desde la perspectiva de la persona con discapacidad.

Esta percepción también fue evaluada en una investigación adelantada por la Escuela de Medicina y Ciencias de la Salud de la Universidad del Rosario (2014) en la búsqueda |de la comprensión de imaginarios, mitos y estereotipos sobre la sexualidad de personas con discapacidad intelectual. Los investigadores, encontraron que en sus familias los términos más usados para referirse a la persona con discapacidad son el "niño", el "enfermo", el "discapacitado mental", "retardado" o "disminuido". También encontraron una polarización entre la visión como niños eternos y asexuados o como seres hipersexuales que deben contar con control y regulación externa de su sexualidad. Estas representaciones sociales hacen que decisiones relacionadas con el derecho al matrimonio, la vida en pareja y la patria potestad de los hijos sean percibidas como totalmente alejadas de las posibles realidades de las personas con discapacidad. En relación con la interdicción y su influencia en esferas íntimas de la vida de los interdictos, estos autores mencionan:

"Constituye una figura jurídica [...] que se estableció como medida de protección patrimonial de las personas con discapacidad intelectual y que hoy se aplica de manera indiscriminada a todas las esferas personales y privadas de las personas declaradas interdictas" (Escuela de Medicina y Ciencias de la Salud, 2014: 5).

Con respecto a la percepción de sí mismos, algunos autores han encontrado en el análisis de las narrativas que mujeres con discapacidad intelectual no se reconocen a sí mismas como seres sexuales (Fitzgerald y Withers, 2011). Sienten que el entorno familiar y los profesionales en salud les prohíben tener actividad 
sexual, lo que genera que perciban que son otros quienes regulan y controlan la afectividad y la forma de expresión del amor, entre otros factores.

Desde la experiencia de ser madre con discapacidad intelectual, por ejemplo, algunas mujeres narran como algunos familiares les sugirieron abortar, muchas manifiestan sentir miedo de perder la custodia de sus hijos, reportan tener experiencias negativas con el personal de salud que atiende el parto en el momento en el que revelan su diagnóstico o dificultades para comprender la información del entorno (Hoglund y Larsson, 2013). Se trata de un sistema de salud y de educación que no está preparado para que las personas con discapacidad asuman roles de maternidad o paternidad, puesto que culturalmente se asume que no están en capacidad de hacerlo.

Otro estudio muestra cómo para jóvenes con discapacidad intelectual es percibido como incorrecto hablar de sexo, sintiéndose en una posición de poder inferior en la que se deben seguir las opiniones de otros (Sullivan et al., 2013: 3460). Adicionalmente, perciben el control que tienen las demás personas sobre sus relaciones, a pesar de lo cual ponen de manifiesto su deseo de entablar relaciones afectivas y sexuales cercanas y de calidad. Estos aspectos también fueron destacados en las narrativas de jóvenes en otros estudios (Rushbrooke et al., 2014), en los que se enuncia que las relaciones afectivas son reguladas por la familia, la escuela o los proveedores de servicios asistenciales. No hay un claro límite de la privacidad personal que parece más bien ser un aspecto amputado. Al respecto vale la pena citar la perspectiva de una persona con discapacidad intelectual, quien se siente empoderada en el tema de derechos:

"People think that they can rule you because you've got a disability... 'You can't choose the colour that you like. As well as your boyfriend you can't pick'. I say, 'Hang on there you! Who do you think you're talking to?' Doesn't mean that I get me support from support staff. You can choose who I love and who I like, and you pick the man for me (Georgia)" (Rushbrooke et al., 2014: 537)'.

Investigaciones como la de Rojas et al. (2014) han puesto de manifiesto la importancia de la sexualidad en tanto aspecto constitutivo del ser humano, que se encuentra presente de manera independiente a constituirse como una persona etiquetada con discapacidad intelectual, el interés por tener una pareja como un aspecto crucial del proyecto de vida, o el deseo de amar, casarse y tener una familia, que en algunas ocasiones se ve obstaculizado por las concepciones ligadas a procedimientos legales. Por ejemplo, muestran en una de las narrativas esta disyuntiva:

“Luna: My father doesn’t think that in the future I will be ready to live with a partner, I don't know, he sees it as being difficult for me.

Susana: Why?

Luna: That is what I ask myself all the time. Well because he is here, he thinks that perhaps I am not capable of living with my partner (...) In other words, he is really on top of me. Ok! He is my legal guardian, my father, I love him a lot but I don't know why he doesn't allow me to take that step. I have told him many times that I am 37 years old" (Rojas et al., 2014) $)^{2}$.

\footnotetext{
1. Traducción propuesta: La gente cree que puede gobernarte porque tienes una discapacidad tú no puedes escoger el color que te gusta del mismo modo que no puedes escoger tu novio. Yo digo: Espérate ahí, ¿con quién crees que estás hablando? No significa que el hecho de que consiga apoyo de los profesionales implique que tú puedas elegir a quien puedo amar, quien me gusta y que escojas el hombre para mí. 2. Traducción propuesta:

Luna: Mi padre no cree que en el futuro pueda estar lista para vivir con una pareja, no sé, lo ve como difícil para mí.
} 
Como se explicó previamente, en Colombia la normativa relega a la familia el lugar de curador principal, sin interés en un entrenamiento previo con relación a las decisiones asistidas, la interdependencia ni la autonomía, lo que deja a la merced de las representaciones sociales y valores culturales la forma y los temas de la vida cotidiana en los que el curador incide o toma decisiones por la persona interdicta, haciendo que esta práctica se convierta en una estrategia usada por las familias para ejercer control sobre las decisiones más íntimas como la sexualidad y la afectividad (Ramírez et al., 2015).

Las tensiones aquí plasmadas no son más que aristas de un fenómeno que hemos abordado desde lo político hasta lo personal. Se trata así de una clara muestra de la necesidad de un cambio estructural que transite por una coherencia normativa con un enfoque de derechos, por el acceso a la información en salud sexual y reproductiva y que desemboque en un trabajo fuerte por derribar los estereotipos y representaciones sociales sobre la dependencia y falta de capacidad de toma de decisiones de las personas con discapacidad que se alejan de respetar su autonomía y dignidad.

\section{De la interdicción a la interdependencia y el apoyo}

El pasar de un modelo de sustitución de la capacidad legal e interdicción a un modelo de toma de decisiones con apoyo implica, más allá de un cambio legislativo y judicial, un cambio en la concepción de la individualidad y la independencia como centrales para el desarrollo personal, y reemplazándolos por posturas comunitarias de afiliación y dependencia. La idea de plantear la toma de decisiones con apoyo nace de la premisa de que todas las personas somos dependientes de otros por el simple hecho de vivir en comunidad, a partir de allí la dependencia se convierte en una característica de la interacción social y comunitaria del ser humano.

MacIntyre (2001) plantea la necesidad de reconocer la vulnerabilidad, la aflicción y la dependencia como rasgos constitutivos del ser humano, a partir de posturas feministas, de movimientos sociales y de la relación madre-hijo como paradigma de las relaciones morales ${ }^{3}$. Para este autor, la filosofía moderna se ha centrado en enaltecer la autonomía individual y su capacidad para la toma de decisiones independientes dejando de lado virtudes de reconocimiento y dependencia. Lo anterior conlleva que desde visiones filosóficas el sujeto independiente sea igualado a un sujeto racional, solitario y sin afiliación a estructuras sociales. Este tipo de postura nos invita a "recuperar lo más humano de aquello que nos hace humanos: la necesidad del otro, el reconocimiento de ese otro, el compartir la interioridad, la sensibilidad y la simpatía por el otro" (Balaguer y Blanch, 2011: 4).

Balaguer y Blanch (2011) ponen en relieve el hecho de que es un error igualar autonomía con independencia, ya que para ellos la autonomía requiere necesariamente de dependencia y, por qué no, interdependen-

\footnotetext{
Susana: ¿Por qué?

Luna: Eso es lo que me pregunto todo el tiempo. Bien porque él está aquí, él piensa que tal vez no soy capaz de vivir con mi pareja (...). En otras palabras, él está realmente encima de mí. jok! Él es mi tutor legal, mi padre, lo amo mucho, pero no sé por qué no me permite dar ese paso. Le he dicho muchas veces que tengo 37 años de edad.

3. Maclntyre (2001: 23) realiza la anotación de que este postulado se basa en el trabajo de Virginia Held, pero no relaciona una obra puntual.
} 
cia, debido a que una característica innegable del ser humano es que es un ser social que convive en comunidad y que nace en un estado de indefensión y vulnerabilidad, el cual logra superar de forma más tardía en comparación con otras especies animales. Por lo cual, para Maclntyre (2001) garantizar las condiciones básicas para las personas con discapacidad deja de ser un interés particular para convertirse en universal asociado a la idea del bien común.

El concepto de interdependencia toma relevancia en este contexto, considerando que puede constituirse como una posible alternativa que, lejos de ser impuesta desde la academia, ha sido vivenciada por los actores del movimiento de vida independiente. Implica entonces el reconocimiento de un aspecto característico del ser humano que trasciende el escenario de la discapacidad: somos indefensos, vulnerables y constantemente necesitamos de otros. Además, puede posicionarse como una estrategia de toma de decisiones coherente con los planteamientos evocados en directrices legales internacionales como la Convención y que convoque a pensar en el respeto por la dignidad humana y la posibilidad de autodeterminación, a través de modelos de toma de decisiones con apoyo, los cuales propician la creación de redes solidarias de acompañamiento y soporte afectivo y social.

A pesar de que han pasado varias décadas desde el desarrollo de este concepto, son pocas las investigaciones que documentan las voces de las personas con discapacidad respecto a esta ruta. No obstante, la investigación del sudafricano Leshota (2015) rescata algunas narrativas que pueden esclarecer lo que implica este concepto para las personas con discapacidad. Al respecto es relevante traer parte de una conversación que surge en dicha investigación:

"Paul: 'So you mean non-disabled people depend on you in some instances!'

Jack: 'Absolutely. Yes they do. When there is no light in the house my wife and my children, for example, rely on me to navigate the room [...] Can you imagine how much time, perhaps money as well, are saved in not having to flip through the telephone directory with every call that goes out? Clearly you need us in some instances and we need you sometimes to navigate these ungodly surrounding with steps and holes all over. So I think we do not entirely depend on you and you do not entirely depend on us. It is not correct to say you are entirely independent from us. And to want to live independently from each other would be a travesty of our sociability as humans"4.

Esta posición hace referencia a la importancia de vivir en comunidad que en el contexto cultural africano está particularmente arraigada. Tiene que ver con la idea de compartir con otros, con un sentimiento de participación y pertenencia mutua que no desconoce las diferencias individuales.

Es así como podemos decir que en la toma de decisiones, tanto personales como públicas, el concepto de interdependencia implica pensar en modelos de toma de decisiones que evoquen este sentido comunitario

\footnotetext{
4. Traducción propuesta:

Jack: Ese podría ser otro extremo [ser independiente de otros]... yo pienso que se hace cada vez más claro que necesitamos de otros y así podemos llamarnos a nosotros mismos interdependientes.

Paul: Lo que quieres decir es que las personas no-discapacitadas dependen de ti de algún modo.

Jack: Totalmente. Ellos dependen de mí. Cuando no hay luz en la casa mi esposa y mis hijos, por ejemplo, confían en mí para transitar por el cuarto (...), ¿puedes imaginar cuánto tiempo, quizás dinero también, nos ahorramos en el hecho de no tener que ir hacia el directorio telefónico con cada llamada que sale? Claramente ustedes nos necesitan a nosotros en algunos aspectos y nosotros necesitamos de ustedes para transitar estos espacios con pasos y huecos por todas partes. Entonces yo pienso que no dependemos completamente de ustedes y ustedes no dependen completamente de nosotros. No es correcto decir que ustedes son totalmente independientes de nosotros. Y querer vivir independientemente de otros sería una parodia de nuestra sociabilidad como seres humanos (...).
} 
de ayudarnos unos a otros, leyendo a esos otros desde sus potencialidades y necesidades humanas; siendo el fin último el cambio de paradigma que ve la discapacidad de forma negativa, para sustituirlo por una nueva visión en la cual el lugar de enunciación de las personas se plante como sujetos políticos.

En este punto, se juega lo personal y lo privado como concepto de subversión que implica un poder de ruptura, de trasgresión y de invención (Lamus, 2009). Un ejemplo de prácticas subversivas, más allá de la connotación negativa que pueda tener este término en Colombia, es el movimiento social self-advocacy ${ }^{5}$ en el Reino Unido, el cual es reconocido por su lucha respecto al cese de prácticas como la esterilización por motivos de discapacidad intelectual. Este movimiento invita a las personas con discapacidad a hablar de sus experiencias sexuales y afectivas abiertamente, partiendo del reconocimiento a la sexualidad como un derecho, y tomando fuerza en países como Suiza, Norteamérica, Reino Unido, Australia y algunos de Europa en las últimas décadas (Docherty et al., 2015). Este tipo de prácticas convocan la noción de que lo personal es político, entendido aquello denominado persona como un objeto de poder (en términos de censura y represión), al mismo tiempo que se convierte en eje de subversión y de resistencia para los movimientos sociales (Parrondo, 2009: 107).

Desde la proximidad del sujeto y su esfera personal se entretejen las relaciones de interdependencia, reconocimiento e identificación que generan lazos sociales, y agrupación de intereses y posteriores movimientos sociales y políticos que generan cambios en la estructura en la que nos vemos inmersos. Es necesario dejar de ver lo personal como lo individual, independiente y propio para comenzar a entenderlo como una realidad "extrasocial" que se instaura a partir del discurso del otro y genera la construcción de identidades (Parrondo, 2009: 107).

Vergara (2012: 60) plantea que todas las prácticas sociales se caracterizan por tener un mínimo de organización e interdependencia, que genera y propicia la participación de las personas en ellas y que, posteriormente, se transformarán en bienes internos para las personas como lo son el dinero y el reconocimiento. En este sentido, todas las actividades que se generan en el marco de las comunidades sociales se caracterizan por un grado de interdepencia que garantiza su desarrollo, en el que la autonomía no se puede ver como un proceso de individualidad, sino como el producto de prácticas comunitarias. Por lo tanto, plantear la capacidad legal como un proceso de interdependencia implica una concepción del ser humano como un ser social inmerso en una comunidad y cuya autonomía se crea a partir de su relación con el otro. En estos términos, los modelos de toma de decisiones con apoyo dan cuenta de modelos sociales y comunitarios que buscan transgredir la lógica de la individualidad, para crear nuevos paradigmas de comunidades solidarias en los cuales las personas con discapacidad se convierten en un grupo garante de la accesibilidad a mecanismos justos de participación para todos, sin excepción, ya que este tema no solo recae en el derecho privado que tienen las personas con discapacidad a decidir sobre sus vidas, salud y sexualidad, en términos de si tener o no hijos, o realizarse procedimientos quirúrgicos, sino en procesos de justicia social para poblaciones históricamente invisibilizadas y marginadas.

5. Podría traducirse como auto-abogacía. 


\section{Referencias bibliográficas}

Balaguer, A. y Blanch, J. (2011): "Deconstruir la discapacidad para repensar la autonomía: propuestas para una pedagogía de la interdependencia”. En XII congreso internacional de la teoría de la educación, Universidad de Barcelona, Barcelona.

Benavides, Á. (2013): Modelos de capacidad jurídica: una reflexión necesaria a la luz del art.12. De la Convención internacional sobre los derechos de las personas con discapacidad. Madrid: Universidad Carlos III De Madrid.

Carlson, I. (2010): The faces of intellectual disability. Indiana: Indiana University Press.

Colombia (2017): Proyecto de ley "No más interdicción para las personas con discapacidad" (en línea). <http:// www.insor.gov.co/descargar/boletinprensa04_2017.pdf> acceso el 1 de noviembre de 2016.

Colombia. Ley 1346 de 2009, de 3 de julio, por medio de la cual se aprueba la "Convención sobre los Derechos de las personas con Discapacidad", adoptada por la Asamblea General de la Naciones Unidas el 13 de diciembre de 2006, Diario Oficial, 31 de julio de 2009, núm. 47427, pp. 54.

Colombia. Ley 1306 de 2009, de 05 de junio de 2009, por la cual se dictan normas para la protección de personas con discapacidad mental y se establece el régimen de la representación legal de incapaces emancipados, Diario Oficial, de 5 de junio de 2009, núm. 47.371 (en línea). <http://www.icbf.gov.co/cargues/avance/docs/ ley_1306_2009.htm>, acceso 25 de noviembre de 2016.

Colombia. Sentencia T-740/14, [MP Luis Ernesto Vargas Silva] de la Corte Suprema de Justicia, Sala Novena de Revisión, de 3 de octubre de 2014 (en línea). <http://www.corteconstitucional.gov.co/RELATORIA/2014/T-740-14. htm>, acceso el 25 de noviembre de 2016.

Colombia. Sentencia T-131/14, [MP María Victoria Calle], Corte Suprema de Justicia, Sala Plena, de 11 de marzo de 2014 (en línea). <http://www.corteconstitucional.gov.co/RELATORIA/2014/C-131-14.htm> acceso el 25 de noviembre de 2016.

Colombia. Ley 57 de 1887, Código Civil Colombiano, Diario Oficial, de 1887 (en línea). <http://www.alcaldiabogota.gov.co/sisjur/normas/Norma1.jsp?i=39535>, acceso 1 de noviembre de 2016.

Cuenca, P. (2012): El sistema de apoyo en la toma de decisiones desde la Convención Internacional sobre los Derechos de las Personas con Discapacidad: principios generales, aspectos centrales e implementación en la legislación español (en línea). <https://dialnet.unirioja.es/servlet/articulo?codigo=4104539> acceso 10 de junio de 2017.

Docherty, D. et al. (2015): Sexuality and relationships in the lives of people with intellectual disabilities: standing in my shoes. Philadelphia: Jessica Kingsley Publishers.

Escuela de Medicina y Ciencias de la Salud. (2014): Derechos sexuales y reproductivos de las personas con discapacidad intelectual: ¿Está preparada nuestra sociedad?. Bogotá: Universidad del Rosario.

Fernández de Bujan, A. (2012): Derecho privado romano. Madrid: lustel S.A.

Fitzgerald, C. y Withers, P. (2011): “I don't know what a proper woman means': what women with intellectual disabilities think about sex, sexuality and themselves”. British Journal Of Learning Disabilities, 41 (1): 5-12.

Herazo, D. (2015): Pautas de interacción y narrativas de las madres acerca del hijo con discapacidad cognitiva en relación con la sexualidad y proyecto de vida. Bogotá: Pontificia Universidad Javeriana. 
Hoglund, B. y Larsson, M. (2013): Struggling for motherhood with an intellectual disability-a qualitative study of women's experiences in Sweden. Midwifery, 29 (6): 698-704.

Inclusion International (2014): Informe mundial sobre el derecho a decidir. Londres: University of East London.

Jaime, J. (2015): La Esterilización Forzosa de PCD a través de los procesos de Interdicción: Una doble vulneración de Derechos Humanos y Fundamentales (en línea). <https://www.minjusticia.gov.co/Portals/0/RUNDIS/ ESTERILIZACION\%20FORZOSA\%20DE\%20PCD\%20A\%20TRAVES\%20DE\%20LOS\%20PROCESOS\%20 DE\%20INTERDICCION.pdf>, acceso 21 de junio de 2017.

Lamus, D. (2009): Movimiento feminista o movimiento de mujeres en Colombia (en línea). <http://revistas.unab. edu.co/index.php?journal=sociojuridico\&page=article\&op=view\&path \%5B \%5D =1321\&path\%5B $\% 5 \mathrm{D}=1286>$ acceso el 25 de noviembre de 2016.

Leshota, P. (2015): "From dependence to interdependence: Towards a practical theology of disability". HTS Teologiese Studies/Theological Studies, 71 (2): 1-9.

López, M. et al. (2010): "Programa de Intervención Psicosexual en Personas con Discapacidad Intelectual”. Educación y diversidad, 4 (2): 75-92.

Lorenzo, R. et al. (2009): Capacidad Jurídica y Discapacidad (Un estudio del derecho privado comparado a la luz de la Convención internacional de derechos de personas con discapacidad). Madrid: Observatorio Estatal de la Discapacidad.

Macintyre, A. (2001): Animales racionales y dependientes. Barcelona: Paidos.

McConnell, D. (2012): “The right to marriage and family”. Journal of intellectual disability research, 56 (7): 655.

Medina, J. et al. (2009): Nuevo régimen de protección legal a las personas con discapacidad mental: antecedentes, análisis y trámite legislativo - ley 1306 de 2009. Bogotá: Universidad Del Rosario.

Organización de las Naciones Unidas, ONU (2016): Observaciones finales sobre el informe inicial de Colombia: Comité sobre los Derechos de las Personas con Discapacidad (en línea). <http://docstore.ohchr.org/SelfServices/FilesHandler.ashx?enc=6QkG1d\%2fPPRiCAqhKb7yhsiZZNrtQsqlapJ5RB16sOGbABEB1GCpxOsNgAj Gfi\%2b3bz9dSJDuD\%2bhgnRmlwPeMHtZbhHsj3D4FpJ8XvrovNgznRYIGHiqFZ5xI4wQSBsKCy>, acceso 5 de junio de 2017.

Organización de las Naciones Unidas, ONU (2006): Convención Internacional sobre los Derechos de las Personas con Discapacidad. Nueva York: ONU.

Palacios, A. y Romañach, J. (2006): El modelo de la diversidad: la bioética y los derechos humanos como herramientas para alcanzar la plena dignidad en la diversidad funcional (en línea). <https://e-archivo.uc3m.es/ bitstream/handle/10016/9899/diversidad.pdf?sequence=1\&isAllowed=y> acceso 15 de noviembre de 2016.

Parrondo, E. (2009): “Lo personal es político”. Trama y fondo: revista de cultura, 27 (1): 105-110.

Ramírez, M. et al. (2015): ¿Amores im(posibles)? Historias narradas y actuadas por hombres y mujeres en situación de discapacidad. Medellín: Universidad de Antioquia.

Rojas, S. et al. (2014): "My great hope in life is to have a house, a family and a daughter': relationships and sexuality in intellectually disabled people". British journal of intelectual disabilities, 44 (1): 56-62.

Rushbrooke, E. et al. (2014): "The experiences of intimate relationships by people with intellectual disabilities: a qualitative study". Journal of applied research in intellectual disabilities, 27 (6): 531-541. 
Stoffelen, J. et al. (2013): "Homosexuality among people with a mild intellectual disability: an explorative study on the lived experiences of homosexual people in the Netherlands with a mild intellectual disability". Journal of Intellectual Disability Research, 57 (3): 257-267.

Sullivan, F. et al. (2013): “Touching people in relationships': a qualitative study of close relationships for people with an intellectual disability". Journal of Clinical Nursing, 22 (1): 3456-3466.

Toboso, M. y Arnau, S. (2008): "La discapacidad dentro del enfoque de capacidades y funcionamientos de Amartya Sen”. Revista Iberoamericana de Filosofía, Política y Humanidades, 10 (20): 64-94.

Valencia, L. (2014): Breve historia de las personas con discapacidad: de la opresión a la lucha por sus derechos (en línea). <http://www.rebelion.org/docs/192745.pdf>, acceso 22 de octubre de 2016.

Vergara, L. (2012): Macintyre, una propuesta de ética plausible (en línea). <https://repository.javeriana.edu.co/ handle/10554/3909>, acceso 10 de junio de 2017. 